\title{
Regelmässig essen und auf den Ernährungstyp achten
}

Ulrike von Blarer Zalokar hat sich auf die Diätetik spezialisiert und betrachtet die Ernährung vor allem aus der Sicht der Chinesischen Medizin. Auf das Essen zu achten ist ihrer Meinung nach ein wichtiger Teil eines gesunden Lebens.

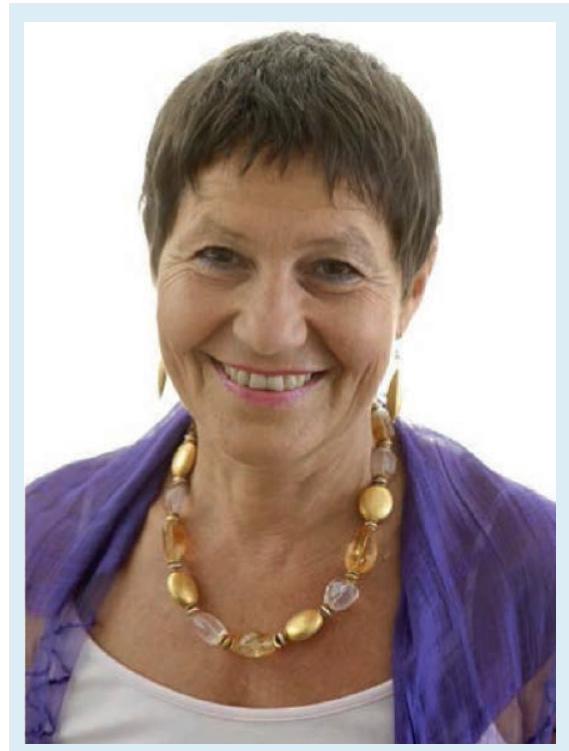

\section{Einfach gut essen}

Gesunde Ernährung ist wichtig und gleichzeitig auch schwierig. Standardrezepte gibt es nicht, denn jedem schmeckt etwas anderes und der Stoffwechsel ist auch nicht bei allen gleich. Zeit also, sich dem Thema Essen im Expertengespräch mit Ulrike von Blarer Zalokar differenziert und mit mehr Genuss zu widmen. Wer sich richtig ernähren möchte, sollte sich selber beobachten. Ärzte können sich einiges bei der Chinesischen Medizin abschauen - Köche, Hausfrauen und Hausmänner übrigens auch.

\section{Geht man den Leuten mit dem Ge- rede über ungesunde Ernährung nicht fürchterlich auf die Nerven? Es stresst doch ungemein, wenn man sich vorstellt, dass $70 \%$ der Frauen, die älter als 40 Jahre sind, angeblich Übergewicht haben. Warum lässt man die Leute nicht essen?}

Ulrike von Blarer Zalokar: Es ist ein grosses Problem, dass wir in Bezug auf die Ernährung sehr einseitig denken. Man glaubt, dass entweder alles besonders vitalstoffreich sein müsse oder sonstige spezielle Inhaltsstoffe enthalten solle. Oder man überlegt sich, was man weglassen muss, etwas, das man aus irgendwelchen Gründen nicht darf, oder etwas, von dem man denkt, es mache dick. Eine Zeit lang wurde Olivenöl propagiert, dann lag das Gewicht auf den Kohlehydraten, auf die man achten musste. Die Ernährungsmoden wechseln tatsächlich sehr schnell. Essen ist zum Aufwand geworden und sollte doch etwas ganz Normales und Tagtägliches sein.

Ich denke, dass etwas in der Art der Ernährung nicht stimmt, sobald etwas weggelassen werden muss. Etwas wegzulassen kann bei Leuten helfen, die schon etwas «dicker» sind und sich schon länger falsch ernährt haben. Wenn Übergewicht entstanden ist, hat unsere denaturierte Nahrung sicherlich einiges dazu beigetragen.

\section{Denaturierte Nahrung durch das Kochen und durch die industrielle Verarbeitung?}

Ja, denaturiert durch die industrielle Herstellung, bei der man etwa dem Getreide viele Vitalstoffe wegnimmt und nur noch die Kohlehydrate zur Verfügung stellt. Damit wird die Ernährung einseitig, und durch Einseitigkeit entstehen viel schneller gesundheitliche Probleme. Der Mensch ist ein Gewohnheitstier und isst jeden Tag gerne das Gleiche. Wir lieben es, wenn wir jeden Morgen unser gleiches Brot kriegen, z.B. Ruchbrot, das bereits denaturiert ist. Zum Mittag essen wir fast täglich Teigwaren, die viel Weizenweissmehl enthalten. Zum z'Vieri gibt es Kuchen oder sonst eine Süssigkeit, die wieder Weizen enthält. Dann kommt das Abendessen, und dazu haben wir oft wieder Brot, und das neben den Teigwaren auf dem Teller - also schon wieder gute Portionen Weizenweissmehl.

Die grössten Schwierigkeiten entstehen dadurch, dass wir Menschen zu wenig abwechslungsreiche Nahrung zu uns nehmen. Dadurch kann es eher $\mathrm{zu}$ gesundheitlichen Entgleisungen kommen.

\section{KARGER}

Fax +4976145207 14 
Das ist eine Abkehr von dem eher natürlichen Ernährungsverhalten des Menschen, bei dem er abwechslungsreich essen musste, weil es in der Natur nicht immer alles gab?

Ich glaube gar nicht, dass man früher so abwechslungsreich gegessen hat - eigentlich könnten wir heute abwechslungsreicher essen als jemals zuvor in der Geschichte der Menschheit. Ich habe mir in jungen Jahren den Spass gemacht, mich möglichst von Produkten zu ernähren, die ich in der Natur gefunden habe. Das war gar nicht so einfach, denn es bleiben einem immer sehr ähnliche Produkte, die jahreszeitmässig höchstens ein bisschen variieren. Im Winter gab es übrigens gar nichts, was man in der Natur finden und essen konnte.

\section{Was haben Sie im Sommer gegessen?}

Zum Beispiel Wiesenbrokkoli, das Wiesenschaumkraut oder Löwenzahn. Aus Brennnesseln habe ich eine Suppe gemacht. Aus Himmelschlüssel und Sauerampfer kann man einen Salat machen. Hauptsächlich gibt es also Gemüse, später im Jahr auch noch Nüsse. Wenn es um Eiweiss geht, wird es schon schwierig, sich ganz aus der Natur zu ernähren. Jagen bin ich nicht gegangen, Insekten wie Käfer sind gewöhnungsbedürftig, aber geröstete Heuschrecken habe ich einmal probiert, und die schmecken eigentlich lecker. Ich habe mich auch schlau gemacht, wie man Maden weiterverarbeitet, aber es bei der Theorie belassen.

Das war also keine valable Lösung für die Ernährungsprobleme unserer Gesellschaft.

Auf keinen Fall. Man muss den Genuss wieder zurück zum Essen bringen, das ist ein Teil der Lösung. Wenn die Freude am Essen verloren geht und sobald Frust eintritt, gibt es immer das gleiche Problem. Der Stoffwechsel funktioniert anders und nicht mehr so, wie er soll. Dann setzt man eher Gewicht an. Das ist der Grund für den Jo-Jo-Effekt, unter dem Leute, die mit ihrem Übergewicht kämpfen, bei ihren ewigen Diäten leiden.

\section{Der Stress, den sich die Leute wegen des Essens machen, schlägt sich dann auf den Stoffwechsel nieder?}

Ja, und generell auch auf die ganze Psyche. Das führt dann zu Situationen, in denen man ein oder zwei Tage gar nichts und dann wieder wie wahnsinnig isst. Eine Lösung bietet da die Chinesische Medizin, die einen sehr vernünftigen Ansatz hat. Sie sagt, dass unsere Verdauungsenergie eine regelmässige Nahrungszufuhr braucht. In dem Moment, in dem ich hungere, schwäche ich meine Verdauungsenergie. Wenn ich zu viel esse, schwäche ich sie, und wenn ich

\section{Ulrike von Blarer Zalokar}

hat 1985 die Heilpraktikerschule Luzern (www.heilpraktikerschule.ch) und in der Folge die HPS Klinik ( $w w w . h p s k l i n i k . c h)$ gegründet. Beide Institutionen leitet sie zusammen mit ihrem Mann Peter von Blarer. Sie unterrichtet Akupunktur, Diätetik West-TCM, Phytotherapie West-TCM und Shiatsu, also diejenigen Fächer, mit denen sie als Heilpraktikerin auch behandelt. Momentan steht die Vergrösserung der Heilpraktikerschule Luzern an. Mehr und grössere Räume sind in einem Neubau geplant; die Grundsteinlegung sollte im Herbst 2013 erfolgen.

Unter anderem hat sie ein Kochbuch, «EssenZ aus der Küche», mit 148 Rezepten unter Berücksichtigung der Chinesischen Medizin und der neuesten wissenschaftlichen Ernährungsforschung veröffentlicht. $\mathrm{Zu}$ beziehen ist es unter: www.heilpraktikerschule.ch. in sehr unterschiedlichen Zeitabständen esse, schwäche ich sie wiederum. Sobald die Verdauungsenergie geschwächt ist, setzt man einfach mehr Fett an.

\section{Das ganze System kommt aus dem Rhythmus. Sind denn drei Mahlzeiten genug oder zu wenig?}

Nach der Chinesischen Medizin soll die Ernährung so eingestellt sein, dass dreimal am Tag essen ausreicht. Das Frühstück sollte so gestaltet sein, dass man nachher vier bis fünf Stunden keinen Hunger hat. Dann kommt das Mittagessen, das bis zum Abendessen vorhalten sollte.

\section{Funktioniert das bei Ihnen auch?}

Ja, seit ich erwachsen bin, läuft das bei mir automatisch so. Das passt zu meinem Verdauungstyp, der ja von Mensch zu Mensch unterschiedlich ist. Ich bin eine Person, die schnell Hunger bekommt, wenn ich kein Öl zu mir nehme, also keine Fettstoffe bekomme. Für mich ist es ideal, wenn ich zum Frühstück Quark mit Öl, Haselnussmus oder Sesammus oder Butter, ein Vollkorn-Roggensauerteigbrot mit Quark und selbst hergestellte Marmelade nehme, die wenig Zucker enthält. So halte ich wunderbar bis zum Mittag durch. Bei meinen Patienten stelle ich das Gleiche fest: Diejenigen, die schnell Hunger bekommen, essen zu wenig Fett und zu viel Zucker und Kohlehydrate.

\section{Wie sieht ein ideales Frühstück aus?}

Das ist von Mensch zu Mensch sehr verschieden. Wenn jemand einen schnellen Zuckerabfall in seinem Körper hat, muss er darauf achten, dass er möglichst wenig zuckerhaltige 
Produkte zu sich nimmt. Er muss sich mit Marmelade, Honig oder gewissen Früchten, wie Bananen, zurückhalten. Dann sollte das Frühstück eher auf Nüsse ausgerichtet sein. Sesam- oder Haselnusspaste auf einer dünnen Scheibe Vollkorn-Roggensauerteigbrot. Roggen hat weniger Kohlehydrate und Zuckerkettung als Weizen und ist deshalb für solche Verdauungstypen besser. Dazu passt viel Quark, der mit etwas Öl angereichert sein sollte. Auch das Frühstücksei oder Speck mit Ei sind geeignet.

\section{Und wie finde ich eigentlich heraus, was für mich die richtige Ernährung ist?}

Da hilft das «metabolic typing», mit dem der Stoffwechseltyp bestimmt wird. Ich erkenne den Typ bei meinen Patienten relativ schnell und einfach, wenn sie mir z.B. berichten, sie bräuchten unbedingt Zwischenmahlzeiten, hätten schnell einen Blutzuckerabfall und müssten etwas Süsses nachschieben, damit sie überhaupt durchhalten. Solche Patienten werden von Getreidenahrung wie Spagetti oder Pizza eher müde und fühlen sich nach dem Essen nicht wirklich vital. Dort merke ich, dass sie eine eiweissreichere Ernährung brauchen, und wenn sie die Ernährung umstellen, kommen sie dann problemlos plötzlich mit einer längeren Pause zwischen den Mahlzeiten aus.

Wie wirkt sich das aus? Gibt es Gewichtsverluste, bessere Laune und ein gesteigertes Wohlgefühl?

Das bessere Wohlgefühl ist sehr schnell da. Sie fühlen sich vitaler und - manche sagen - weniger benebelt und freier im Kopf. Manche sind fröhlicher und kommen sich tatkräftiger vor. Bei der Entwicklung des Gewichts kommt es immer auf die persönliche Situation an. Ich habe Patienten, die

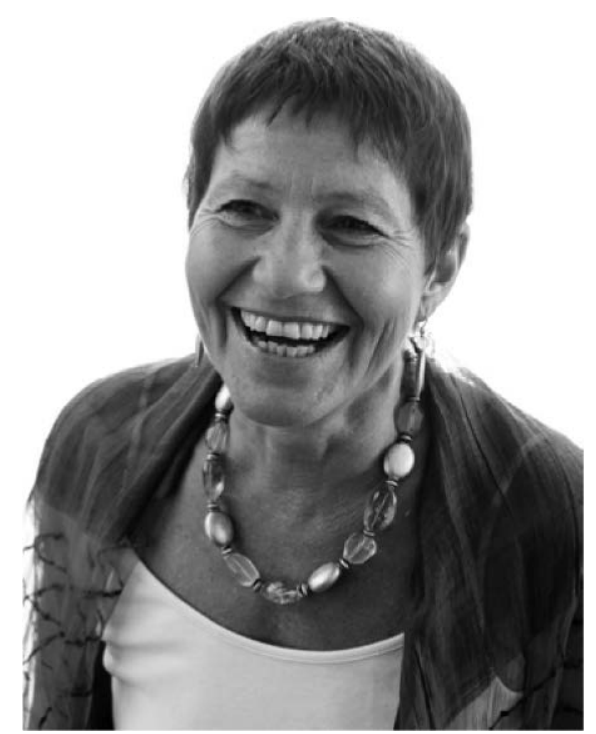

Sinn. Ausserdem isst man in Amerika zu einseitig - zu wenig gute Fette und zu viele Kohlehydrate.

\section{Wie erkennt man, dass die Ver- dauungsenergie geschwächt ist?}

Sobald ein Mensch einen zu weichen Stuhl hat, ist die Mitte geschwächt. Ich gebe meinen Patienten immer die Aufgabe mit, auf den Stuhl zu achten. Man kennt das ja, wenn man mal etwas zu viel Alkohol getrunken hat, auswärts gegessen hat dann geht alles durcheinander. In der Chinesischen Medizin heisst es, dass dann feuchte Hitze im Körper gar nicht die Absicht haben abzunehmen, aber dann wirklich an Gewicht verlieren. Was man nicht vergessen darf, ist, dass es beim Gewicht auch immer um die Menge geht, die jemand isst. Die kann man nur langsam reduzieren. Wir wissen, dass sich der Magen mit der Menge dehnt, und wenn er weniger bekommt, bildet er sich auch zurück, was allerdings seine Zeit braucht.

\section{Funktionieren Tricks, wie kleinere Teller, um Leute zu weniger Essen zu bringen? Es gibt ja die These, dass in Amerika die Leute auf- grund der grösseren Portionen dicker geworden sind.}

In Amerika spielen aus der Sicht der Chinesischen Medizin aber auch noch andere Themen eine Rolle. In Amerika trinkt man eiskaltes Wasser - selbst in den Wein kommt ein Eiswürfel rein. In der Chinesischen Medizin gehen wir davon aus, dass unsere Mitte, also die Verdauungsenergie, nicht mehr richtig arbeitet, wenn wir sie abkühlen. Die Nahrung wird nicht vollständig umgewandelt, sondern bleibt in der Umwandlung stecken und wird - chinesisch-medizinisch gesprochen - als Schleim abgelagert, also als Fett im westlichen ist und er nicht mehr richtig funktioniert. Am besten ist das am Stuhl zu erkennen, und das ist so einfach, dass jeder in der Lage ist, täglich etwas über seinen Gesundheitszustand zu erfahren.

Das Problem ist aber, bei jemandem das Interesse für die eigene Ernährung, die Verdauung und die Nahrungsmittel, die man zu sich nimmt, zu wecken. Was in ihnen enthalten ist, weiss niemand mehr. Es will sich auch kaum jemand damit beschäftigen; dazu fehlt heute einfach vielen die Zeit.

\section{Da ist doch erstaunlich, wenn man bedenkt, wie viel Aufmerksamkeit dem Thema Essen gewidmet wird - es ist doch in der Gesellschaft dauernd präsent.}

Stimmt, das Thema beschäftigt, aber hauptsächlich die Nahrungsmittelindustrie. Die alte Tradition des Wissens um die Ernährung und die Zubereitung ist abgerissen. Heute haben viele nicht mehr das Wissen und nehmen sich auch nicht die Zeit, um zu kochen, und viele haben sie auch nicht. Wer den ganzen Tag arbeitet, kann am Abend kaum noch lange in der Küche stehen. Ich habe das gesehen, als ich die Koch- 
kurse noch selber gegeben habe. Da kamen junge Frauen, die konnten Blumenkohl und Kohlrabi nicht unterscheiden.

\section{Was gibt es für Möglichkeiten, um das Kochen oder die Ernährung wieder interessant und zum Thema zu machen?}

Das ist schwierig. In England hat es Jamie Oliver ja zum Teil geschafft, mit Spass an der schnellen Küche Leute wieder an den Herd zu bringen. Wichtig ist, dass es rasch gehen muss. Mehr als eine Viertelstunde liegt für viele bei der Vorbereitung nicht drin - also noch weniger als die Zeit, die man braucht, um Spaghetti zu kochen. Für die Kochkurse habe ich Rezepte zusammengestellt, die eine Alternative $\mathrm{zu}$ den üblichen Spaghetti sind und trotzdem wenig Zeit brauchen. So sind Hirse, Quinoa oder Buchweizen viel gesünder, und sie lassen sich in etwa derselben Zeit zubereiten wie Spaghetti. Das überzeugt die Leute, und sie können rasch eine Alternative zu den Teigwaren-Kohlehydraten auf den Tisch bringen.

Eine Rolle spielt auch, dass der Geschmack nicht geschult ist. Das muss schon im Kindesalter beginnen. Man muss Kinder sehr früh an eine abwechslungsreiche Ernährung gewöhnen und sie mit speziellen Gerichten am Familientisch verwöhnen. Immer nur Teigwaren, Kartoffeln und weisser Reis sind überhaupt nicht sinnvoll. Selbst wenn sie sich anfänglich sträuben, etwas mit einem unbekannten Geschmack zu essen, haben sie sich beim vierten oder fünften Mal daran gewöhnt.

Dadurch kann man den Kindern helfen, dass sie ihr Spektrum an Nahrungsmitteln nicht einengen, sondern vergrössern. Und vielleicht, wenn sie später als Erwachsene in die Ferien fahren, essen sie dann nicht dasselbe, was sie von daheim kennen, sondern lassen sich auf die kulinarischen Abenteuer ein, abseits von Pizza oder Fast-Food-Imbissen.

\section{Wieso kommen Leute mit Ernährungsproblemen zu Ihnen und suchen Hilfe?}

Es gibt viele Patienten, die selber merken, dass bei ihnen mit der Ernährung etwas nicht stimmt; etwa, wenn sie ständig Koliken haben, unter Übergewicht leiden bzw. Rheuma oder Arthrose haben. Sehr häufig hilft ein Blick auf ihre Ernährung und darauf, wie häufig sie essen, um zu erkennen, was sie eigentlich krank macht.

In der Chinesischen Medizin kommt der Ernährung eine zentrale Rolle zu. So gibt es dort ein Prinzip, das wir hier eigentlich gar nicht verstehen: Zuerst sollte via Ernährung geheilt werden, bevor mit Kräutern oder Akupunktur unterstützt wird. Angewendet auf einen Rheumapatienten heisst das, dass ich darauf achte, ob er z.B. viele Nachtschattengewächse isst. Die verstärken nach den Vorstellungen der Chinesischen Medizin das Kälte-Nässe-Bi, wie Rheuma chinesisch-medizinisch genannt wird. Also müssen Rheumapatienten Tomaten, Peperoni, Auberginen und Kartoffeln weglassen. Eine weitere diätetische Massnahme ist es, das Kälte-Nässe-Bi mithilfe von Nahrungsmitteln, die Nässe eliminieren, auszuleiten. Dazu gehören Linsen, Kichererbsen, Pilze oder Bohnen. Die stehen meistens nicht auf dem Küchenplan. Bauen die Patienten sie ein, dann geht es ihnen oft besser.

\section{Wie gross ist die Bereitschaft der Leute, ihr Essverhalten umzustellen?}

Das ist stark typenabhängig und hat natürlich auch mit dem Leidensdruck zu tun. Es gibt Leute, die gehen nach Hause, entsorgen die für sie ungesunden Sachen aus dem Küchenschrank und besorgen sich die für sie passenden Lebensmittel. Bei anderen ist es oft ein langwieriger Prozess, der 2-3 Jahre dauern kann. Es ist ohnehin im Laufe des Lebens notwendig, die Ernährung den sich ständig verändernden Umständen und dem Stoffwechsel anzupassen. Gemäss der Chinesischen Medizin verliert die Verdauungsenergie beim alternden Menschen an Kraft, wie etwa bei Frauen in der Menopause.

Viele Frauen werden dann hitziger, was sich häufig nebst Wallungen auch an einer Trockenheit im Körper bis hin zum trockenen Stuhl zeigt, eben als Verstopfung. In jungen Jahren litten diese Frauen oft unter Kälte wie kalten Händen und Füssen und eventuell unter einem sehr weichen, feuchten Stuhl. Wenn eine Frau vor der Menopause sehr warm oder eher trocknend - beides sind chinesischmedizinische Eigenschaften von Nahrungsmitteln - essen musste, braucht sie nun etwas kühlere und befeuchtende Sachen. Beispielsweise Nahrungsmittel, die mehr Flüssigkeit führen und öliger sind, wie etwa Salat, Avocados, Algen, Keimlinge, gute Öle und Früchte, wenn sie sie verträgt. Scharfes Curry, Chili oder Knoblauch oder Rotwein wird dann eher nicht mehr gut vertragen; diese Nahrungsmittel steigern die Hitze zusätzlich und sollten daher weggelassen oder reduziert werden. Sehr Yang-lastiges Fleisch wie Lammfleisch oder Rindfleisch - unabhängig von der Zubereitung, geschweige, wenn es noch grilliert wurde - kann bei Hitze-Patienten auch problematisch sein. Alternativen sind dann eher Kaninchen-, Hühner- und Putenfleisch oder Fisch. Es sind meist kleine Veränderungen in der Ernährung, aber die Frauen merken schnell, dass es ihnen besser geht - die Hitzezustände und -wallungen bleiben aus und die Verdauung funktioniert dann auch wieder. 
Müssen sich Männer im Laufe des Lebens auch in der Ernährung ihrem Alter und den Veränderungen anpassen?

Das, was für Frauen gilt, gilt auch für die Männer. Wenn ein Mann zu mir kommt und er feststellt, dass er weniger gut schläft, sich die Verdauung geändert hat und er in der Nacht schwitzt, ist das auch ein Zustand, in dem sich das Gleichgewicht der Yinund Yang-Energie im Körper verändert hat. Das heisst, er ist hitziger geworden und hat ein relatives YangÜbergewicht, weilsein Yinabgesunken ist. Das ist genau das gleiche Thema wie bei der Frau, und er muss die beiden Energien im Körper wieder in ein Gleichgewicht bringen. Es kann aber auch genau umgekehrt sein, dass ein Mensch im Alter zu frieren beginnt. Wichtig ist, dass man sich in allen Lebensphasen beobachtet und dann entsprechend reagiert.

\section{Was muss man bei der Ernährung von Kindern aus der Sicht der Chinesischen Medizin beachten?}

Bei Babys ist das Stillen ein grosses Thema. Dabei spielt auch die Frage, wie sich die Mutter ernährt, eine grosse Rolle. Viele wissen z.B. nicht, dass der Fettgehalt in der Muttermilch davon abhängt, wie viele Fette oder Öle sie zu sich nimmt. Nimmt die
Mutter gute Fette und Öle zu sich, hat das Baby etwas davon, nimmt sie schlechte, dann leider auch. Deshalb ist es so wichtig für das Kind, was die Mutter während der Stillzeit isst.

\section{Was halten Sie von vorgefertigter Babykost?}

Wenn eine Mutter nicht stillen kann, ist z.B. entsprechend aufbereitete Milch, die die Kinder vertragen, ein Segen - damit bringt man die Babys über die Runden. Wenn dann der Übergang zur festeren Nahrung ansteht, ist es immer wieder die Frage, ob man fertige Produkte kauft oder selber etwas zubereitet. Das frisch Zubereitete ist nach wie vor von Vorteil, denn vorgefertigte Babynahrung ist meist zu süss. Am besten ist es, die Kinder nicht an den süssen Geschmack zu gewöhnen. Die Süsse der Karotte oder auch des Fenchels ist ausreichend.

Was ich immer wieder feststelle, ist, dass stillende Mütter auch noch anfangen, die Baby-Nahrung selber immer frisch zu kochen, und in einen Wahnsinnsstress kommen, sodass auch ihre Muttermilch zurückgeht. In chinesischen Familien ist es üblich, dass die Frau während des ersten Lebensjahrs des Kindes eine Arbeitshilfe bekommt; die Grossmutter oder eine andere Verwandte geht zur Hand.
Bei uns werden die Frauen sehr allein gelassen.

\section{Welche Ernährung brauchen \\ Jugendliche, wenn sie pubertieren?}

In dieser Zeit macht der Mensch einen wahnsinnigen Prozess durch. Dann verändert sich ja auch das TagNacht-Verhältnis. Pubertierende beginnen die Nächte durchzumachen. Die Nacht, in der die Entgiftung des Körpers stattfinden sollte, wird zum Tag. Anstatt den Körper regenerieren zu lassen, wird gegessen, getrunken und geraucht. Auch verändert sich in dieser Zeit der Serotoninhaushalt; daher glaube ich, dass es Jugendlichen gut tun würde, wenn sie mehr tryptophan- und serotoninreichere Nahrung essen würden. Ich habe das Gefühl, dass sie dann über diese wahnsinnig starken Veränderungen schneller und leichter kommen würden.

Das meiste Tryptophan enthalten Baumnüsse; aber auch Hülsenfrüchte wie Sojabohnen, Fisch oder Cashewkerne sind zu empfehlen. Ganz interessant ist auch Kakao. Er hilft bei zahlreichen Schlafproblemen nicht nur in der Pubertät. Das heisst, Eltern dürfen ihren Kindern den Kakao gönnen, wenn sie ihnen etwas Gutes tun wollen. Mit Kakao, das muss ich natürlich auch sagen, meine ich Kakao in Form von mindestens 70\%iger Schokolade.

Interview: Oliver Klaffke 